\title{
Identifying Reaction Functions in Differential Oligopoly Games ${ }^{1}$
}

\author{
Luca Lambertini $^{2}$ and Andrea Mantovani ${ }^{3}$
}

September 2004

\footnotetext{
${ }^{1}$ We thank Rabah Amir, Roberto Cellini, Ulrich Doraszelski and the seminar audience at Université Catholique de Louvain and 31st EARIE Conference (Berlin, September 2-5, 2004) for useful comments and suggestions. The usual disclaimer applies.

${ }^{2}$ Department of Economics, University of Bologna, Strada Maggiore 45, I-40125 Bologna, Italy, lamberti@spbo.unibo.it; and ENCORE, Faculty of Economics \& Econometrics, University of Amsterdam, Roetersstraat 11, WB1018 Amsterdam, The Netherlands.

${ }^{3}$ CORE, Université Catholique de Louvain, 34 Voie du Roman Pays, B-1348, Louvain-la-Neuve, Belgium, mantovani@core.ucl.ac.be and Department of Economics, University of Bologna, Strada Maggiore 45, I-40125 Bologna, Italy, mantovan@spbo.unibo.it
} 


\begin{abstract}
We investigate the issue of strategic substitutability/complementarity in differential games. We prove that instantaneous best replies exist if Hamiltonian functions are multiplicative in the control variables. Otherwise, if the Hamiltonians are addively separable w.r.t. controls, a dominant strategy emerges for each player. In this case, however, imposing stationarity on the differential equations of states, one can still identify best replies at the steady state, which is ruled out by definition in static games.
\end{abstract}

JEL classification: C73, D43, D92, L13.

Keywords: complementarity/substitutability, differential games, reaction functions 


\section{Introduction}

The issue of super-/submodularity has been investigated mostly in static games, and refers to the slope of reaction functions in the (stage) game, as initially pointed out by Bulow, Geanakoplos and Klemperer (1985). Recently, the concept of economic complementarity has emerged as a leading theme of research. The analysis has been focused on games with strategic complementarities and their use in industrial economics (Vives, 1990; Milgrom and Roberts, 1990 and Amir, 1996) and in comparative statics analysis (Milgrom and Shannon, 1994).

A potential development of this discussion consists in investigating whether the same properties can be reconstructed in a differential game, and to what extent. ${ }^{1}$ The static game approach obviously rules out a priori the possibility of intertemporal strategic substitutability/complementarity. On the contrary, by using a differential game approach we can enlarge the class of games showing strategic complementarities by considering firms' interaction along the equilibrium path.

To the best of our knowledge, only Jun and Vives (2004) carry out theoretical research on intertemporal strategic complementarity/substitutability. They compare steady states of open loop and stable closed-loop equilibria in symmetric differentiated duopoly model with adjustment costs. One of the most interesting result is that strategic complementarity of the static price game becomes intertemporal strategic substitutability in the presence of costly production adjustment. ${ }^{2}$

\footnotetext{
${ }^{1}$ For an overview of differential game theory and applications, see See Başar and Olsder (1982, 1995²), Mehlmann (1988) and Dockner et al. (2000). For differential oligopoly games, see Cellini and Lambertini (2003).

${ }^{2}$ Zulehner (2003) tests the presence of dynamic strategic interaction in the dynamic
} 
In the present paper, we propose two different models based on the capital accumulation dynamics introduced by Ramsey (1928) and Solow (1956) - Swan (1956), respectively. In both cases the open-loop Nash equilibrium coincides with the closed-loop memoryless equilibrium, and hence the former is subgame perfect. First we consider the Ramsey model reformulated as an oligopoly game, where firms transform unsold output into additional productive capacity. The model admits instantaneous best reply functions describing strategic interaction among firms at any instant from the outset to the steady state. Secondly, and more interestingly, we consider the SolowSwan model, where firms invest in order to accumulate capacity, and then operate at full capacity at any time. In this model, first order conditions do not produce instantaneous best reply functions, since optimal controls depend only on state variables at any time during the game, which therefore is solved in dominant strategies. In such a case, however, it is still possible to evaluate strategic interaction in steady state, by imposing the stationarity condition on the kinematic equations describing the behaviour of state and control variables.

The plan of the paper is as follows. Section 2 introduces the concept of reaction function along the equilibrium path. The Ramsey model is described in Section 3, while Section 4 investigates the Solow model. Section 5 concludes the paper.

random access memory (DRAM) industry. She estimates a dynamic oligopoly model incorporating learning-by-doing and spillovers, showing that firms take into account the intertemporal strategic effect of their contemporaneous output decisions on their rivals' future output decision. Jarmin (1994) raises the same question by looking at the early rayon industry and found empirical evidence of dynamic strategic behavior. 


\section{Preliminaries}

Consider a generic differential game, played over continuous time, with $t \in$ $[0, \infty){ }^{3}$ The set of players is $\mathbb{P} \equiv\{1,2, \ldots N\}$. Moreover, let $x_{i}(t)$ and $u_{i}(t)$ define, as usual, the state variable and the control variable pertaining to player $i$. Assume there exists a prescribed set $\mathcal{U}_{i}$ such that any admissible action $u_{i}(t) \in \mathcal{U}_{i}$. The dynamics of player $i$ 's state variable is described by the following:

$$
\frac{d x_{i}(t)}{d t} \equiv \dot{x}_{i}(t)=f_{i}(\mathbf{x}(t), \mathbf{u}(t))
$$

where $\mathbf{x}(t)=\left(x_{1}(t), x_{2}(t), \ldots x_{N}(t)\right)$ is the vector of state variables at time $t$, and $\mathbf{u}(t)=\left(u_{1}(t), u_{2}(t), \ldots u_{N}(t)\right)$ is the vector of players' actions at the same date, i.e., it is the vector of control variables at time $t$. That is, in the most general case, the dynamics of the state variable associated with player $i$ depends on all state and control variables associated with all players involved in the game. The value of the state variables at $t=0$ is assumed to be known: $\mathbf{x}(0)=\left(x_{1}(0), x_{2}(0), \ldots x_{N}(0)\right)$.

Each player has an objective function, defined as the discounted value of the flow of payoffs over time. The instantaneous payoff depends upon the choices made by player $i$ as well as its rival, that is:

$$
\pi_{i} \equiv \pi_{i}(\mathbf{x}(t), \mathbf{u}(t))
$$

Player $i$ 's objective is then, given $u_{j}(t), j \neq i$ :

$$
\max _{u_{i}(\cdot)} J_{i} \equiv \int_{0}^{\infty} \pi_{i}(\mathbf{x}(t), \mathbf{u}(t)) e^{-\rho t} d t
$$

\footnotetext{
${ }^{3}$ One could also consider a finite terminal time $T$. The specific choice of the time horizon is immaterial to the ensuing analysis, provided that terminal conditions are appropriately defined.
} 
subject to the dynamic constraint represented by the behaviour of the state variables, $(1), u_{i}(t) \in \mathcal{U}_{i}$ and initial conditions $\mathbf{x}(0)=\left(x_{1}(0), x_{2}(0), \ldots x_{N}(0)\right)$.

The Hamiltonian of player $i$ is:

$$
\begin{aligned}
\mathcal{H}_{i}(\mathbf{x}(t), \mathbf{u}(t)) \equiv & e^{-\rho t}\left[\pi_{i}(\mathbf{x}(t), \mathbf{u}(t))+\lambda_{i i}(t) \cdot f_{i}(\mathbf{x}(t), \mathbf{u}(t))+\right. \\
& \left.+\sum_{j \neq i} \lambda_{i j}(t) \cdot f_{j}(\mathbf{x}(t), \mathbf{u}(t))\right]
\end{aligned}
$$

where $\lambda_{i j}(t)=\mu_{i j}(t) e^{\rho t}$ is the co-state variable (evaluated at time $t$ ) that firm $i$ associates with the state variable $x_{j}(t)$.

The interesting property, in the present perspective, is summarised by the second cross-derivative w.r.t. controls:

$$
\frac{\partial^{2} \mathcal{H}_{i}}{\partial u_{i} \partial u_{j}}
$$

Two cases are possible:

- If

$$
\frac{\partial^{2} \mathcal{H}_{i}}{\partial u_{i} \partial u_{j}} \neq 0
$$

then the first order condition (FOC)

$$
\frac{\partial \mathcal{H}_{i}}{\partial u_{i}}=0
$$

yields the instantaneous best reply function of player $i$ against any admissible choice of player $j$ at any time $t$, and

$$
\operatorname{sgn}\left(\frac{\partial^{2} \mathcal{H}_{i}}{\partial u_{i} \partial u_{j}}\right)
$$

is the slope of such best reply function.

- If instead

$$
\frac{\partial^{2} \mathcal{H}_{i}}{\partial u_{i} \partial u_{j}}=0 \forall j \neq i
$$


then the FOC does not yield an instantaneous best reply function. The necessary and sufficient condition for (9) to hold is additive separability of the Hamiltonian of player $i$ w.r.t. control variables. In this situation, it must be nonetheless true that the expression $\partial \mathcal{H}_{i} / \partial u_{i}$ contains the costate variables. Hence, in order to solve for the equilibrium path of $u_{i}$, one has to take the derivative of $(7)$ w.r.t. $t$. This yields:

$$
\dot{u}_{i}=z_{i}\left(\dot{\lambda}_{i i}, \dot{\lambda}_{i j}, \dot{x}_{i}, \dot{x}_{j}\right)
$$

where $\dot{x}_{i}, \dot{x}_{j}$ are given by state equations (1), and the dynamics of the costate variables $\lambda_{i i}$ and $\lambda_{i j}$ comes from the costate equations:

$$
-\frac{\partial \mathcal{H}_{i}}{\partial x_{i}}-\sum_{j \neq i} \frac{\partial \mathcal{H}_{i}}{\partial u_{j}} \frac{\partial u_{j}^{*}}{\partial x_{i}}=\dot{\lambda}_{i i}-\rho \lambda_{i i}
$$

If (11) contains $\left(u_{i}, u_{j}\right)$, then, by substitution, we will observe $\dot{u}_{i}=$ $w_{i}\left(u_{i}, u_{j}\right)$. Imposing $\dot{u}_{i}=0$, one obtains $u_{i}^{*}=v_{i}\left(u_{j}\right)$ representing the best reply against the choice of $j$ in the steady state equilibrium.

The difference between the two cases lies in the fact that while in the first case we observe an instantaneous reaction function characterisng the optimal behaviour of player $i$ at any time during the game, in the second case we only observe player $i$ 's best reply at the steady state equilibrium, while $i$ 's optimal behaviour during the transition to the steady state can be characterised in terms of states and costates only, regardless of any player $j$ 's control. This amounts indeed to saying that along the path to the steady state each player has a dominant strategy. This discussion is summarised by:

Remark 1 If player $i$ 's Hamiltonian is additively separable w.r.t. controls, then $\partial^{2} \mathcal{H}_{i} / \partial u_{i} \partial u_{j}=0$ and each player $i$ has a dominant strategy at every instant. 
To better illustrate these issues, we propose two examples based upon differential oligopoly games investigated in the existing literature.

\section{The Ramsey model}

Here, we revisit the differential game analysed in Cellini and Lambertini (1998). Consider a market where $N$ single-product firms offer differentiated products over $t \in[0, \infty)$. At any time $t$, the inverse demand function for variety $i$ is (see Spence, 1976):

$$
p_{i}(t)=A-B q_{i}(t)-D \sum_{j \neq i} q_{j}(t)
$$

where $D \in[0, B]$ is the symmetric degree of substitutability between any pair of varieties. If $D=B$, products are completely homogeneous; if $D=0$, strategic interaction disappears and firms are independent monopolists. The direct demand function obtains by inverting (12):

$$
q_{i}(t)=\frac{1}{B+D(N-1)} \cdot\left\{A-\frac{[B+D(N-2)] p_{i}(t)}{B-D}+\frac{D}{B-D} \sum_{j \neq i} p_{j}(t)\right\} .
$$

Producing any variety $i$ requires physical capital $k$, accumulating over time to create capacity. At any $t$, the output level is $y_{i}(t)=f\left(k_{i}(t)\right)$, with $f^{\prime} \equiv$ $\partial f\left(k_{i}(t)\right) / \partial k_{i}(t)>0$ and $f^{\prime \prime} \equiv \partial^{2} f\left(k_{i}(t)\right) / \partial k_{i}(t)^{2}<0$.

A reasonable assumption is that $q_{i}(t) \leq y_{i}(t)$, that is, the level of sales is at most equal to the quantity produced. Excess output is reintroduced into the production process yielding accumulation of capacity according to the following process (Ramsey, 1928):

$$
\frac{d k_{i}(t)}{d t}=f\left(k_{i}(t)\right)-q_{i}(t)-\delta k_{i}(t)
$$


where $\delta$ denotes the rate of depreciation of capital. In order to simplify further the analysis, suppose that unit variable cost is constant and equal to zero. The cost of capital is represented by the opportunity cost of intertemporal relocation of unsold output. Firm $i$ 's instantaneous profits are

$$
\pi_{i}(t)=p_{i}(t) q_{i}(t)
$$

Firm $i$ maximizes the discounted flow of its profits:

$$
J_{i}=\int_{0}^{\infty} e^{-\rho t} \pi_{i}(t) d t
$$

under the constraint (14) imposed by the dynamics of the state variable $k_{i}(t)$. Notice that the state variable does not enter directly the objective function. It can be assumed, alternatively, that all firms behave as either quantitysetters or price setters. Hence, the control variable is either $q_{i}(t)$ when all firms are Cournot agents, or $p_{i}(t)$ in the case where firms adopt a Bertrand behaviour.

\subsection{Cournot competition}

When firms compete in quantities, substitute (12) in (16) to get the relevant objective function of firm $i$ :

$$
J_{i}=\int_{0}^{\infty} e^{-\rho t} q_{i}(t) \cdot\left[A-B q_{i}(t)-D \sum_{j \neq i} q_{j}(t)\right] d t
$$

which must be maximised w.r.t. $q_{i}(t)$, under (14). The corresponding Hamiltonian function is:

$$
\begin{aligned}
\mathcal{H}_{i}(\cdot)= & e^{-\rho t} \cdot\left\{q_{i}(t)\left[A-B q_{i}(t)-D \sum_{j \neq i} q_{j}(t)\right]+\right. \\
& +\lambda_{i i}(t)\left[f\left(k_{i}(t)\right)-q_{i}(t)-\delta k_{i}(t)\right]+ \\
& \left.+\sum_{j \neq i} \lambda_{i j}(t)\left[f\left(k_{j}(t)\right)-q_{j}(t)-\delta k_{j}(t)\right]\right\},
\end{aligned}
$$


where $\lambda_{i i}(t)=\mu_{i i}(t) e^{\rho t}$ and $\lambda_{i j}(t)=\mu_{i j}(t) e^{\rho t} ; \mu_{i i}(t)$ and $\mu_{i j}(t)$ are the co-state variable associated respectively to state variables $k_{i}(t)$ and $k_{j}(t)$.

We focus on the closed-loop solution, which provides an equilibrium characterized by subgame perfection. The necessary conditions for a path to be optimal are: ${ }^{4}$

$$
\begin{aligned}
& \text { (i) } \frac{\partial \mathcal{H}_{i}(\cdot)}{\partial q_{i}(t)}=A-2 B q_{i}(t)-D \sum_{j \neq i} q_{j}(t)-\lambda_{i}(t)=0, \\
& (i i)-\frac{\partial \mathcal{H}_{i}(\cdot)}{\partial k_{i}(t)}-\sum_{j \neq i} \frac{\partial \mathcal{H}_{i}(\cdot)}{\partial q_{j}(t)} \frac{\partial q_{j}^{*}(t)}{\partial k_{i}(t)}=\frac{\partial \lambda_{i i}(t)}{\partial t}-\rho \lambda_{i i}(t), \\
& \left(i i^{\prime}\right)-\frac{\partial \mathcal{H}_{i}(\cdot)}{\partial k_{j}(t)}-\sum_{m \neq j} \frac{\partial \mathcal{H}_{i}(\cdot)}{\partial q_{m}(t)} \frac{\partial q_{m}^{*}(t)}{\partial k_{j}(t)}=\frac{\partial \lambda_{i j}(t)}{\partial t}-\rho \lambda_{i j}(t),
\end{aligned}
$$

along with the transversality conditions:

$$
\lim _{t \rightarrow \infty} e^{-\rho t} \lambda_{i j}(t) \cdot k_{j}(t)=0 \forall j=1,2,3 \ldots, i, \ldots N .
$$

From $(19-i)$, we note that

$$
\frac{\partial^{2} \mathcal{H}_{i}(\cdot)}{\partial q_{i}(t) \partial k_{j}(t)}=0
$$

which entails that the feedbacks that would characterise the closed-loop solution are null. From $(19-i)$ we also obtain the instantaneous best reply function:

$$
q_{i}^{*}(t)=\frac{A-D \sum_{j \neq i} q_{j}(t)-\lambda_{i i}(t)}{2 B}
$$

Note that, since the best reply is independent of $\lambda_{i j}(t)$ for all $j \neq i$, the game admits the solution $\lambda_{i j}(t)=0$ for all $j \neq i$ and the co-state equation (19ii') is indeed redundant. Therefore, the closed-loop solution collapses onto the open-loop solution and the game belongs to the so-called class of perfect games, where the open-loop equilibrium is a degenerate closed-loop one. ${ }^{5}$

\footnotetext{
${ }^{4}$ Second order conditions are also satisfied. They are omitted for brevity.

${ }^{5}$ See Mehlmann (1988, ch. 4), Dockner et al. (2000, ch. 7) and Cellini and Lambertini (2001).
} 
Now focus on identifying a best reply function. Expression (22) is a standard Cournot reaction function, except for the presence of the co-state variable $\lambda_{i i}(t)$, which, however, only affects the intercept, but not the slope:

$$
\frac{\partial q_{i}^{*}(t)}{\partial q_{j}(t)}=-\frac{D}{2 B}
$$

As usual in Cournot games, quantities are strategic substitutes (resp., complements) when goods are demand substitutes (resp., complements). This suggests that firms may behave as in the static game, as far as the slope of their best reply functions is concerned. However, before drawing a neat conclusion to this regard, we must proceed to the characterisation of the steady state equilibrium by adopting the open-loop solution concept. The first order conditions on control as well as the transversality conditions are the same as above, while the co-state equation (19-ii) simplifies as follows: ${ }^{6}$

$$
-\frac{\partial \mathcal{H}_{i}}{\partial k_{i}}=\frac{\partial \lambda_{i i}}{\partial t}-\rho \lambda_{i i} \Rightarrow \frac{\partial \lambda_{i i}}{\partial t}=\lambda_{i i}\left[\rho+\delta-f^{\prime}\left(k_{i}\right)\right] .
$$

The function (22) can be differentiated w.r.t. time to get

$$
\frac{d q_{i}}{d t}=\frac{-D \sum_{j \neq i}\left(d q_{j} / d t\right)-d \lambda_{i i} / d t}{2 B}
$$

Thanks to (24), the expression in (25) simplifies as follows:

$$
\frac{d q_{i}}{d t}=\frac{1}{2 B}\left[\left(f^{\prime}\left(k_{i}\right)-\rho-\delta\right) \lambda_{i i}-D \sum_{j \neq i} \frac{d q_{j}}{d t}\right] .
$$

In order to simplify calculations and to obtain an analytical solution, we assume, on the basis of firms' ex ante symmetry, $\sum_{j \neq i} q_{j}=(N-1) q_{i}$, so that $\sum_{j \neq i} d q_{j} / d t=(N-1) d q_{i} / d t$. Thanks to symmetry, in the remainder

\footnotetext{
${ }^{6}$ Henceforth, the indication of time is omitted for the sake of brevity.
} 
we drop the index of the firm. As a further simplification, we also drop the indication of time.

Finally, from (22) we can derive an explicit expression for $\lambda_{i i}$, that, simplified by using symmetry, writes:

$$
\lambda_{i i}=A-[2 B+D(N-1)] q .
$$

We can now easily rewrite (26) as follows:

$$
\frac{d q}{d t}=\left(f^{\prime}(k)-\rho-\delta\right) \cdot \frac{A-[2 B+D(N-1)] q}{2 B+D(N-1)} .
$$

The equilibrium analysis can be carried out by using a phase diagram in the space $\{k, q\}$, which accounts for the stability properties of steady state points (figure 1).

Figure 1: Cournot competition

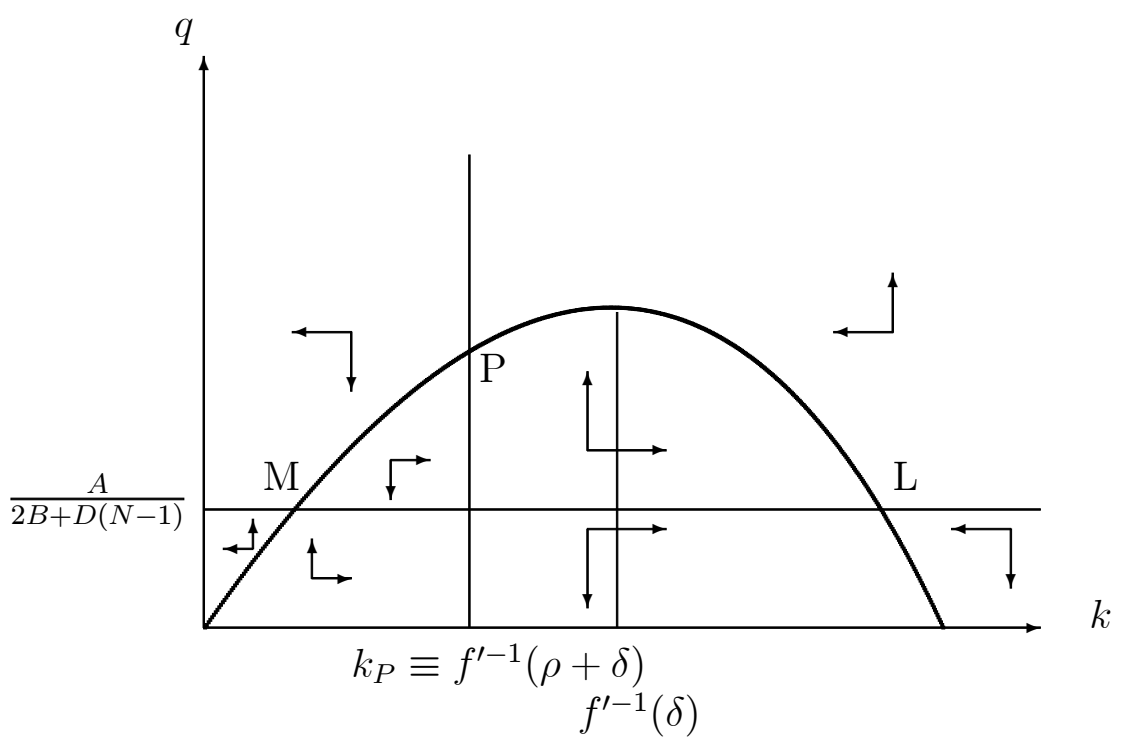

The locus $\dot{q} \equiv d q / d t=0$ is given by $q^{s s}=A /(2 B+D(N-1))$ where superscript ss stands for steady state, and $f^{\prime}(k)=\rho+\delta$ in figure 1 . The 
solution $q^{s s}=A /(2 B+D(N-1))$ denotes the usual equilibrium output we already know from the static literature (see, e.g., Singh and Vives, 1984; Vives, 1985). The two loci partition the space $\{k, q\}$ into four regions, where the dynamics of $q$ is determined by (28), as summarised by the vertical arrows. The locus $\dot{k} \equiv d k / d t=0$ as well as the dynamics of $k$, depicted by horizontal arrows, derive from (14). Steady states, denoted by $M, L$ along the horizontal arm, and $P$ along the vertical one, are identified by intersections between loci.

The situation represented in figure 1 is one out of five possible configurations, due to the fact that the position of the vertical line $f^{\prime}(k)=\rho+\delta$ is independent of demand parameters, while the horizontal locus $q=A /(2 B+$ $D(N-1))$ shifts upwards (downwards) as $A(B, D$ and $N)$ increases. Therefore, we obtain one out of five possible regimes:

[1] There exist three steady state points, with $k_{M}<k_{P}<k_{L}$ (as in Figure 1).

[2] There exist two steady state points, with $k_{M}=k_{P}<k_{L}$.

[3] There exist three steady state points, with $k_{P}<k_{M}<k_{L}$.

[4] There exist two steady state points, with $k_{P}<k_{M}=k_{L}$.

[5] There exists a unique steady state point, corresponding to $P$.

An intuitive explanation for the above taxonomy can be provided, in the following terms. The vertical locus $f^{\prime}(k)=\rho+\delta$ identifies a constraint on optimal capital embodying firms' intertemporal preferences, i.e., their common discount rate. Accordingly, maximum output level in steady state would be that corresponding to (i) $\rho=0$, and (ii) a capacity such that 
$f^{\prime}(k)=\delta$. This would be the golden rule of capacity accumulation. Yet, a positive discounting (that is, impatience) induces producers to install a smaller steady state capacity according to a modified golden rule, much the same as it happens in the well known Ramsey model. On these grounds, define this level of $k$ as the optimal capital constraint, and label it as $\widehat{k}$. When the reservation price $A$ is very large (or $B, D, N$, are low), points $M$ and $L$ either do not exist (regime [5]) or fall to the right of $P$ (regimes [2], [3], and [4]). Under these circumstances, the capital constraint is operative and firms choose the capital accumulation corresponding to $P$. As we will see below, this is fully consistent with the dynamic properties of the steady state points.

Notice that, since both steady state points located along the horizontal locus entail the same levels of sales, point $L$ is surely inefficient in that it requires a higher amount of capital. Point $M$, as already mentioned above, corresponds to the optimal quantity emerging from the static version of the game. It is hardly the case of emphasising that this solution encompasses both monopoly (either when $N=1$ or when $D=0$ ) and perfect competition (as, in the limit, $N \rightarrow \infty){ }^{7}$ In point $M, d \pi_{i} / d q_{i}=0$, that is, the marginal instantaneous profit is nil.

Now we come to the stability analysis of the above system. The joint dynamics of $q$ and $k$, can be described by linearising (28) and (14) around

\footnotetext{
${ }^{7}$ The analysis of dynamic monopoly with capital accumulation dates back to Evans (1924). See Chiang (1992) for a recent exposition of the original model by Evans, as well as later developments.
} 
$\left(k^{s s}, q^{s s}\right)$, to get what follows:

$$
\left[\begin{array}{l}
\dot{k} \\
\dot{q}
\end{array}\right]=\Xi\left[\begin{array}{c}
\left(k-k^{s s}\right) \\
\left(q-q^{s s}\right)
\end{array}\right]
$$

where

$$
\Xi=\left[\begin{array}{cc}
f^{\prime}(k)-\delta & -1 \\
\frac{A-(2 B+D(N-1)) q}{2 B+D(N-1)} f^{\prime \prime}(k) & -\left(f^{\prime}(k)-\rho-\delta\right)
\end{array}\right]
$$

The stability properties of the system in the neighbourhood of the steady state depend upon the trace and determinant of the $2 \times 2$ matrix $\Xi$. In studying the system, we confine to steady state points. The trace of $\Xi$ is $\operatorname{tr}(\Xi)=\rho>0$, whereas the determinant $\Delta(\Xi)$ varies according to the point where it is evaluated. Consider the above taxonomy.

Regime [1]. In $M, \Delta(\Xi)<0$, hence this is a saddle point. In $P, \Delta(\Xi)>0$, so that $P$ is an unstable focus. In $L, \Delta(\Xi)<0$, and this is again a saddle point, with the horizontal line as the stable arm.

Regime [2]. In this regime, $M$ Coincides with $P$, so that we have only two steady states which are both are saddle points. In $M=P$, the saddle path approaches the saddle point from the left only, while in $L$ the stable arm is again the horizontal line.

Regime [3]. Here, $P$ is a saddle; $M$ is an unstable focus; $L$ is a saddle point, as in regimes [1] and [2]. 
Regime [4]. Here, points $M$ and $L$ coincide. $P$ remains a saddle, while $M=L$ is a saddle whose converging arm proceeds from the right along the horizontal line.

Regime [5]. Here, there exists a unique steady state point, $P$, which is also a saddle point.

We can sum up the above discussion as follows. The unique efficient and non-unstable steady state point is $P$ if $k_{P}<k_{M}$, while it is $M$ if the opposite inequality holds. Such a point is always a saddle. Individual equilibrium output is $q_{M}^{s s}=A /(2 B+D(N-1))$ if the equilibrium is identified by point $M$, or the level corresponding to the optimal capital constraint $\widehat{k}$ if the equilibrium is identified by point $P$. The reason is that, if the capacity at which marginal instantaneous profit is nil is larger than the optimal capital constraint, the latter becomes binding. Otherwise, the capital constraint is irrelevant, and firms' decisions in each period are solely driven by the unconstrained maximisation of single-period profits. It is apparent that, in the present setting, firms always operate at full capacity. When optimal output is $q_{M}^{s s}$, per-firm instantaneous profits in steady state are

$$
\pi_{M}^{s s}=\frac{A^{2} B}{[2 B+D(N-1)]^{2}}
$$

while they are

$$
\pi_{P}^{s s}=f(\widehat{k})\{A-[B+D(N-1)] f(\widehat{k})\}
$$

if optimal output is $f(\widehat{k})$. Notice that when the Ramsey equilibrium prevails, firms's behaviour simply consists in setting $q=f(\widehat{k})$, which is indeed a vertical best reply, independent of the behaviour of the rivals, as well as the degree of complementarity/substitutability among products.

The foregoing analysis entails the following: 
Proposition 2 The Ramsey-Cournot game exhibits instantaneous best replies obtained from first order conditions on controls if and only if $q_{M}^{s s} \leq f(\widehat{k})$. In particular:

- if $q_{M}^{s s}<f(\widehat{k})$, then the equilibrium output is $q_{M}^{s s}$, attained through best replies replicating those of the static game;

- if $q_{M}^{s s}=f(\widehat{k})$, then firms attain the equilibrium output following either the quasi-static best replies, or the modified golden rule.

If $q_{M}^{s s}>f(\widehat{k})$, then best replies are vertical and firms follow the modified golden rule.

\subsection{Bertrand competition}

Consider now the alternative setting where firms compete in prices. Also in this case, it can be shown that the open-loop solution is subgame perfect. The demand function is (13), and firm $i$ 's dynamic problem is:

$$
\begin{aligned}
& \max _{p_{i}(t)} J_{i}=\int_{0}^{\infty} e^{-\rho t} p_{i}(t) \cdot\left\{\frac{A(B-D)-[B+D(N-2)] p_{i}(t)+D \sum_{j \neq i} p_{j}(t)}{[B+D(N-1)](B-D)}\right\} d t \\
& \text { s.t. } \dot{k}_{i}(t)=f\left(k_{i}(t)\right)-\delta k_{i}(t)-\frac{A(B-D)-[B+D(N-2)] p_{i}(t)+D \sum_{j \neq i} p_{j}(t)}{[B+D(N-1)](B-D)} .
\end{aligned}
$$

The corresponding Hamiltonian function is now relatively straightforward:

$$
\begin{aligned}
& \mathcal{H}_{i}(\cdot)=e^{-\rho t} \cdot\left\{p_{i}(t) \cdot\left[\frac{A(B-D)-[B+D(N-2)] p_{i}(t)+D \sum_{j \neq i} p_{j}(t)}{[B+D(N-1)](B-D)}\right]+\right. \\
& +\zeta_{i i}(t)\left[f\left(k_{i}(t)\right)-\delta k_{i}(t)-\frac{A(B-D)-[B+D(N-2)] p_{i}(t)+D \sum_{j \neq i} p_{j}(t)}{[B+D(N-1)](B-D)}\right] \\
& \left.+\sum_{j=1}^{N} \zeta_{i j}(t)\left[f\left(k_{j}(t)\right)-\delta k_{j}(t)-\frac{A(B-D)-[B+D(N-2)] p_{j}(t)+D \sum_{m \neq j} p_{m}(t)}{[B+D(N-1)](B-D)}\right]\right\}
\end{aligned}
$$


where $\zeta_{i i}(t)=\theta_{i i}(t) e^{\rho t}$ and $\zeta_{i j}(t)=\theta_{i j}(t) e^{\rho t} ; \theta_{i i}(t)$ and $\theta_{i j}(t)$ are the costate variables associated by firm $i$ to the state variables $k_{i}(t)$ and $k_{j}(t)$, respectively.

By the same procedure as above, one can establish that the FOC on $p_{i}(t)$ yields an instantaneous best reply which (i) depends also on $\zeta_{i i}((t)$; and (ii) is positively (resp., negatively) sloped for substitutes (complements). The FOC of firm $i$ is:

$$
\frac{\partial \mathcal{H}_{i}(\cdot)}{\partial p_{i}(t)}=\frac{A(B-D)+\left[\zeta_{i i}(t)-2 p_{i}(t)\right][B+D(N-2)]+D \sum_{j \neq i} p_{j}(t)}{[B+D(N-1)](B-D)}=0
$$

which yields firm $i$ 's best reply, provided that the market-driven equilibrium prevails in steady state:

$$
p_{i}^{*}(t)=\frac{A(B-D)+\zeta_{i i}(t)[B+D(N-2)]+D \sum_{j \neq i} p_{j}(t)}{2[B+D(N-2)]} .
$$

It can be easily ascertained that the above expression coincides with the standard best reply function of a static game with the same demand structure, except for the presence of the co-state variable $\zeta_{i i}(t) .{ }^{8}$ By taking the partial derivative of $p_{i}^{*}(t)$ w.r.t. $p_{j}(t)$, we obtain:

$$
\frac{\partial p_{i}^{*}(t)}{\partial p_{j}(t)}=\frac{D}{2[B+D(N-2)]}
$$

which is the same slope the best reply function exhibits in the static game. Moreover, since $p_{i}^{b r}(t)$ does not depend on any state variable, the open-loop solution is subgame perfect, and we can proceed to characterise the open-loop version of the co-state equation pertaining to firm $i$ 's capacity: ${ }^{9}$

$$
-\frac{\partial \mathcal{H}_{i}}{\partial k_{i}}=\frac{\partial \theta_{i i}}{\partial t} \Rightarrow \frac{\partial \zeta_{i i}}{\partial t}=\left[\rho+\delta-f^{\prime}\left(k_{i}\right)\right] \zeta_{i i} .
$$

\footnotetext{
${ }^{8} \mathrm{As}$ in the Cournot version of the Ramsey game, also here $\zeta_{i j}(t)=0$ for all $j \neq i$ is an admissible solution.

${ }^{9}$ Again, the indication of time is omitted in the remainder of the section.
} 
On the basis of FOCs, and using the symmetry assumption $\sum_{j \neq i} p_{j}=$ $(N-1) p_{i}$, the necessary and sufficient conditions for the optimal path obtain:

$$
\dot{p} \equiv \frac{d p}{d t}=0 ; \dot{k} \equiv \frac{d k}{d t}=0,
$$

along with the standard transversality condition

$$
\lim _{t \rightarrow \infty} \theta_{i j} k_{j}=0 \forall j=1,2,3 \ldots, i, \ldots N .
$$

The explicit derivation of expressions (39) is omitted for brevity. The locus $\dot{p}=0$ consists of two orthogonal lines along which $f^{\prime}(k)=\rho+\delta$ (as in the Cournot case) and

$$
p^{s s}=\frac{A(B-D)}{2(B-D)+D(N-1)},
$$

respectively. The stability analysis of the price-setting case is qualitatively analogous to the case of quantity-setting behaviour. When (41) identifies a saddle point, individual equilibrium output is

$$
q_{M}^{s s}=\frac{A[B+D(N-2)]}{(2(B-D)+D(N-1))(B+D(N-1))} .
$$

Otherwise, equilibrium output corresponds to the level $f(\widehat{k})$ identified by the optimal capacity constraint $\widehat{k}$ at the Ramsey golden rule $f^{\prime}(k)=\rho+\delta$. In the former case, instantaneous steady state profits per firm are

$$
\pi_{M}^{s s}=\frac{A^{2}(B-D)[B+D(N-2)]}{[2(B-D)+D(N-1)]^{2}[B+D(N-1)]}
$$

while $\pi_{P}^{s s}=f(\widehat{k})\{A-[B+D(N-1)] f(\widehat{k})\}$ as in the Cournot case, if optimal output is $f(\widehat{k})$. In such a case, the equilibrium pricing rule is $p(\widehat{k})=A-f(\widehat{k})[B+D(N-1)]$. This entails the following:

Proposition 3 In the market-driven equilibrium, Bertrand best replies coincide with those observed in the static game. However, if the modified golden rule prevails at equilibrium, best replies in the price space are vertical. 
Propositions 2-3 produce the following ancillary result:

Corollary 4 Under the modified golden rule, best reply functions are always vertical, irrespective of the strategy space.

Both the Cournot and the Bertrand oligopoly games with capital accumulation à la Ramsey admit then instantaneous reaction functions from first order conditions on controls, provided the market-driven equilibrium is selected in steady state. The only difference with respect to the static case consists in the presence of the co-state variable, which however is null at steady state. However, market interaction may well be irrelevant in this dynamic setting. This is precisely what happens when the modified golden rule drives the equilibrium behaviour of firms.

\section{The Solow model}

The market exists over $t \in[0, \infty)$, and is served by $N$ firms producing a homogeneous good. Let $q_{i}(t)$ define the quantity sold by firm $i$ at time $t$. The marginal production cost is constant and equal to $c$ for all firms, and each firm has to build up capacity $k_{i}(t)$ over time through instantaneous investment efforts $I_{i}(t)$, according to the following law of motion à la Solow (1956) - Swan (1956):

$$
\frac{d k_{i}(t)}{d t}=I_{i}(t)-\delta k_{i}(t)
$$

where $\delta \in[0,1]$ is the constant depreciation rate common to all firms. Therefore, in this model, the control variable is $I_{i}(t)$, while capacity $k_{i}(t)$ is the state variable. The kinematic equation of player $i$ 's state variable is unaffected by the state and control variables of rivals. That is, strategic interaction among firms takes place through instantaneous profits only. 
We assume that firms operate with a decreasing returns technology $q_{i}(t)=$ $f\left(k_{i}(t)\right)$, with $f^{\prime} \equiv \partial f\left(k_{i}(t)\right) / \partial k_{i}(t)>0$ and $f^{\prime \prime} \equiv \partial^{2} f\left(k_{i}(t)\right) / \partial k_{i}(t)^{2}<0$. The market demand function at time $t$ is:

$$
p(t)=A-B \sum_{i=1}^{N} f\left(k_{i}(t)\right) .
$$

As shown in Cellini and Lambertini (2001), given that firms do not choose either quantities or prices, the present game produces the same equilibrium outcome irrespective of whether one uses direct or inverse demand functions. On this basis, for simplicity we may assume that firms sell homogeneous goods, as in (45).

The instantaneous cost of investment is $C_{i}\left[I_{i}(t)\right]=b\left[I_{i}(t)\right]^{2} / 2$, with $b>0$, and instantaneous profits are $\pi_{i}(t)=[p(t)-c] f\left(k_{i}(t)\right)-b\left[I_{i}(t)\right]^{2} / 2$. The relevant Hamiltonian for firm $i$ is:

$$
\begin{aligned}
\mathcal{H}_{i}(\cdot)= & e^{-\rho t}\left\{\left[A-B f\left(k_{i}(t)\right)-B \sum_{j \neq i} f\left(k_{j}(t)\right)-c\right] f\left(k_{i}(t)\right)-\frac{b}{2}\left[I_{i}(t)\right]^{2}+\right. \\
& \left.+\lambda_{i i}(t)\left[I_{i}(t)-\delta k_{i}(t)\right]+\sum_{j \neq i} \lambda_{i j}(t)\left[I_{j}(t)-\delta k_{j}(t)\right]\right\} .
\end{aligned}
$$

Necessary conditions for the closed-loop memoryless equilibrium are: ${ }^{10}$

$$
\begin{aligned}
& (i) \frac{\partial \mathcal{H}_{i}(\cdot)}{\partial I_{i}(t)}=0 \Rightarrow-b I_{i}(t)+\lambda_{i i}(t)=0 \Rightarrow I_{i}^{*}(t)=\lambda_{i i}(t) / b \\
& (i i)-\frac{\partial \mathcal{H}_{i}(\cdot)}{\partial k_{i}(t)}-\sum_{j \neq i} \frac{\partial \mathcal{H}_{i}(\cdot)}{\partial I_{j}(t)} \frac{\partial I_{j}^{*}(t)}{\partial k_{i}(t)}=\frac{\partial \lambda_{i i}(t)}{\partial t}-\rho \lambda_{i i}(t) \Rightarrow \\
& \Rightarrow \frac{\partial \lambda_{i i}(t)}{\partial t}=(\rho+\delta) \lambda_{i i}(t)+f^{\prime}\left(k_{i}(t)\right)\left[2 B f\left(k_{i}(t)\right)+B \sum_{j \neq i} f\left(k_{j}(t)\right)-(A-c)\right] \\
& \left(i i^{\prime}\right)-\frac{\partial \mathcal{H}_{i}(\cdot)}{\partial k_{j}(t)}-\sum_{m \neq j} \frac{\partial \mathcal{H}_{i}(\cdot)}{\partial I_{m}(t)} \frac{\partial I_{m}^{*}(t)}{\partial k_{j}(t)}=\frac{\partial \lambda_{i j}(t)}{\partial t}-\rho \lambda_{i j}(t),
\end{aligned}
$$

\footnotetext{
${ }^{10}$ The feedback solution of a similar model, where the instantaneous cost of investment also contains a linear component, is in Reynolds (1987).
} 
with the transversality conditions:

$$
\lim _{t \rightarrow \infty} e^{-\rho t} \lambda_{i i}(t) k_{i}(t)=0 \text { for all } i, j .
$$

Now observe that, on the basis of $(47-i)$, we have: ${ }^{11}$

$$
\frac{\partial I_{j}^{*}}{\partial k_{i}}=0 \text { for all } i, j \text {. }
$$

Moreover, condition $\left(47-i i^{\prime}\right)$, which yields $\partial \lambda_{i j} / \partial t$, is redundant in that $\lambda_{i j}$ does not appear in the first order conditions (47-i) and (47-ii). As before, the closed-loop solution degenerates into the open-loop one.

Now focus on the issue of identifying a best reply function. Condition $(47-i)$ does not contain the control of the other players, i.e. $\partial I_{i}^{*} / \partial I_{j}=0$. Therefore, as it stands, it cannot yield a best reply function for player $i$. This involves the following result:

Lemma 5 At any time during the game, firm i's investment decision is taken on the basis of a dominant strategy.

That is, the optimal investment carried out at any time by any firm is exclusively dictated by the shadow value of an additional unit of capacity. Nevertheless, in the remainder of the section we show that the steady state analysis does convey some information as to the nature of strategic interaction in this game. To this aim, we proceed as follows. Differentiating (47-i) w.r.t. time we obtain:

$$
\frac{\partial I_{i}}{\partial t}=\frac{1}{b} \cdot \frac{\partial \lambda_{i i}}{\partial t}
$$

Then, replace $(47-i)$ into $(47-i i)$, to get the following expression for the dynamics of the costate variable $\lambda_{i i}$ :

$$
\frac{\partial \lambda_{i i}}{\partial t}=b(\rho+\delta) I_{i}+f^{\prime}\left(k_{i}\right)\left[B\left(2 f\left(k_{i}\right)+\sum_{j \neq i} f\left(k_{j}\right)\right)-(A-c)\right],
$$

\footnotetext{
${ }^{11}$ The indication of time is omitted henceforth, for the sake of brevity.
} 
which can be plugged into (50), that rewrites as:

$$
\frac{\partial I_{i}}{\partial t}=(\rho+\delta) I_{i}+\frac{f^{\prime}\left(k_{i}\right)}{b}\left[B\left(2 f\left(k_{i}\right)+\sum_{j \neq i} f\left(k_{j}\right)\right)-(A-c)\right],
$$

To make things simpler, suppose $f\left(k_{i}\right)=k_{i}$. If so, (52) rewrites as:

$$
\frac{\partial I_{i}}{\partial t}=(\rho+\delta) I_{i}+\frac{1}{b}\left[B\left(2 k_{i}+\sum_{j \neq i} k_{j}\right)-(A-c)\right] .
$$

Observe that one can solve $\partial I_{i} / \partial t=0$, so as to obtain:

$$
I_{i}=\frac{1}{b(\rho+\delta)}\left[A-c-B\left(2 k_{i}+\sum_{j \neq i} k_{j}\right)\right]
$$

Still, (53-54) do not contain $I_{j}$, for all $j \neq i$. Therefore, as one could expect in the light of Lemma 5, the optimal (stationary) investment behaviour of firm $i$, described by (54), is determined only by the vector of state variables, irrespective of any firm $j$ 's control. Yet, from the dynamics of the state variable of any player, we know that the equilibrium path of the capacity requires $d k_{j} / d t=0$, yielding:

$$
k_{j}=\frac{I_{j}}{\delta} .
$$

By substituting (55) in (53) we obtain:

$$
\frac{\partial I_{i}}{\partial t}=(\rho+\delta) I_{i}+\frac{1}{b}\left[2 B \frac{I_{i}}{\delta}+B \sum_{j \neq i} \frac{I_{j}}{\delta}-(A-c)\right] .
$$

At this point, imposin stationarity:

$$
\frac{\partial I_{i}}{\partial t}=(\rho+\delta) I_{i}+\frac{1}{b}\left[2 B \frac{I_{i}}{\delta}+B \sum_{j \neq i} \frac{I_{j}}{\delta}-(A-c)\right]=0
$$

yields:

$$
I_{i}^{*}=\frac{\delta(A-c)-B \sum_{j \neq i} I_{j}}{2 B+b \delta(\rho+\delta)}
$$


Hence, we have a negatively sloped best reply function in steady state:

$$
\frac{\partial I_{i}^{*}}{\partial I_{j}}=-\frac{B}{2 B+b \delta(\rho+\delta)} .
$$

Accordingly, we can state the following:

Proposition 6 The Solow game exhibits negatively sloped best reply functions only in the steady state point, where investment efforts behave as strategic substitutes.

We can now complete the exposition of the present model by fully characterising the steady state equilibrium. Invoking symmetry across firms and simplifying, (53) can be rewritten as:

$$
\frac{\partial I}{\partial t}=\frac{1}{b}[b(\rho+\delta) I+B(N+1) k-(A-c)],
$$

with the right hand side being zero at:

$$
I=\frac{A-c-B(N+1) k}{b(\rho+\delta)},
$$

while $d k / d t=0$ at:

$$
k^{s s}=\frac{A-c}{B(N+1)+b \delta(\rho+\delta)} .
$$

By substituting (62) into (61) we get:

$$
I^{s s}=\frac{\delta(A-c)}{B(N+1)+b \delta(\rho+\delta)} .
$$

Using (44) and (53) it is possible to verify that the steady state identifies a saddle point (see Cellini and Lambertini, 2001).

The corresponding per firm instantaneous steady state profits are given by:

$$
\pi^{s s}=\frac{(A-c)^{2}[4 B+b \delta(\delta+2 \rho)]}{2[B(N+1)+b \delta(\rho+\delta)]^{2}} .
$$


A few remarks are in order. The main difference between the Ramsey model on one side and the Solow-Swan model on the other is that the Hamiltonian of each player in the Ramsey game is not additively separable w.r.t. controls, while that appearing in the Solow-Swan game is. Additive separability w.r.t. controls is necessary and sufficient for the first order condition of player $i$ to be independent of any rivals' controls. Therefore, this property ensures that first order conditions do not yield instantaneous best reply functions. If this is the case, then one can impose stationarity on states and, by doing so, derive best replies holding among controls in steady state only.

The latter case describes a game where strategic interaction is so weak that each player can compute the optimal path to the steady state without taking into account the rivals' behaviour. This amounts to saying that each player involved in such a game has a dominant strategy. ${ }^{12}$

\section{Conclusion}

In this paper we have investigated the issue of intertemporal strategic interaction in differential games. We have considered Ramsey and Solow-Swan's growth models, reformulated as oligopoly games. In the Ramsey model, capital accumulation takes place through unsold output (i.e. consumption postponement), while in the Solow model capital accumulation requires a costly investment.

In the first model, intertemporal strategic interaction is almost equivalent to the static one. In fact, the slope of the reaction functions remains the same

\footnotetext{
${ }^{12}$ Several other games characterised by this property can be found in the literature on dynamic advertising or R\&D. See Leitmann and Schmitendorf (1978), Feichtinger (1983) and Cellini and Lambertini (2004), inter alia.
} 
as in static settings, the only difference being represented by the presence of the co-state variable which affects only the intercept. In the second model, first order conditions are independent of rivals' controls, and consequently each player has a dominant strategy at any time during the game. However, after imposing the stationarity condition, we have been able to identify negatively sloped best reply functions in steady state.

The emergence of a dominant strategy is due to the property of additive separability of the Hamiltonian of each player w.r.t. controls. Likewise, in static games, additive separability of the objective function w.r.t. choice variables would indeed entail the existence of a dominant strategy. However, the dynamic analysis allows one to characterise best replies, although in steady state only, precisely by imposing stationarity on the kinematics of state variables, which by definition cannot be done in static games.

\section{References}

[1] Amir, R. (1996), "Cournot Oligopoly and the Theory of Supermodular Games", Games and Economic Behavior, 15, 132-148.

[2] Başar, T. and G.J. Olsder (1982, 1995²), Dynamic Noncooperative Game Theory, San Diego, Academic Press.

[3] Bulow, J. I., Geanakoplos, J. D. and P. D. Klemperer (1985), "Multimarket Oligopoly: Strategic Substitutes and Complements", Journal of Political Economy, 93, 488-511.

[4] Cellini, R. and L. Lambertini (1998), "A Dynamic Model of Differentiated Oligopoly with Capital Accumulation", Journal of Economic Theory, 83, 145-155. 
[5] Cellini, R. and L. Lambertini (2001), "Differential Oligopoly Games where the Closed-Loop Memoryless and Open-Loop Equilibria Coincide", wp 402, Dipartimento di Scienze Economiche, Università degli Studi di Bologna.

[6] Cellini, R. and Lambertini, L. (2003), "Differential Oligopoly Games", in P. Bianchi and L. Lambertini (eds), Technology, Information and Market Dynamics: Topics in Advanced Industrial Organization, Edward Elgar, Cheltenham, pp. 173-207.

[7] Cellini, R. and L. Lambertini (2004), "R\&D Incentives and Market Structure: A Dynamic Analysis", Journal of Optimization Theory and Applications, forthcoming.

[8] Chiang, A. (1992), Elements of Dynamic Optimization, New York, McGraw-Hill.

[9] Dockner, E.J, S. Jørgensen, N. Van Long and G. Sorger (2000), Differential Games in Economics and Management Science, Cambridge, Cambridge University Press.

[10] Evans, G. C. (1924), "The Dynamics of Monopoly", American Mathematical Monthly, 31, 75-83.

[11] Feichtinger, G. (1983), "The Nash Solution of an Advertising Differential Game: Generalization of a Model by Leitmann and Schmitendorf", IEEE Transactions on Automatic Control, 28, 1044-1048.

[12] Jarmin, R. S. (1994), "Learning by Doing and Competition in the Early Rayon Industry", RAND Journal of Economics, 250, 441-454. 
[13] Jun, B. and X. Vives (2004), "Strategic Incentives in Dynamic Duopoly", Journal of Economic Theory, 116, 249-281.

[14] Kamien, M. and I. Zang (2000), "Meet Me Halfway: Research Joint Ventures and Absorptive Capacity", International Journal of Industrial Organization, 18, 995-1012.

[15] Leitmann, G. and W.E. Schmitendorf (1978), "Profit Maximization through Advertising: A Nonzero Sum Differential Game Approach", IEEE Transactions on Automatic Control, 23, 646-650.

[16] Mehlmann, A. (1988), Applied Differential Games, New York, Plenum Press.

[17] Milgrom, P. and J. Roberts (1990a), "Rationalizability, Learning, and Equilibrium in Games with Strategic Complementarities", Econometrica, 58, 1255-1278.

[18] Milgrom, P. and C. Shannon (1994), "Monotone Comparative Statics", Econometrica, 62, 157-180.

[19] Ramsey, F.P. (1928), "A Mathematical Theory of Saving", Economic Journal, 38, 543-59. Reprinted in Stiglitz, J.E. and H. Uzawa (1969, eds.), Readings in the Modern Theory of Economic Growth, Cambridge, MA, MIT Press.

[20] Reynolds, S.S. (1987), "Capacity Investment, Preemption and Commitment in an Infinite Horizon Model", International Economic Review, 28, 69-88.

[21] Singh, N. and X. Vives (1984), "Price and Quantity Competition in a Differentiated Duopoly", RAND Journal of Economics, 15, 546-554. 
[22] Solow, R. (1956), "A Contribution to the Theory of Economic Growth", Quarterly Journal of Economics, 70, 65-94.

[23] Spence, A.M. (1976), "Product Selection, Fixed Costs, and Monopolistic Competition", Review of Economic Studies, 43, 217-236.

[24] Swan, T.W. (1956), "Economic Growth and Capital Accumulation", Economic Record, 32, 334-361.

[25] Vives, X. (1985), "Efficiency of Bertrand and Cournot Equilibria with Product Differentiation", Journal of Economic Theory, 36, 166-175.

[26] Vives, X. (1990), "Nash Equilibrium with Strategic Complementarities", Journal of Mathematical Economics, 19, 305-321.

[27] Zulehner, C. (2003), "Testing dynamic oligopolistic interaction: evidence from the semiconductor industry", International Journal of Industrial Organization, 21, 1527-1556. 